Research Article

\title{
Recognition of Power Equipment Based on Multitask Sparse Representation
}

\author{
Lei Lei, ${ }^{1,2}$ Jian Wu $\mathbb{D}^{1,2}$ Shuhai Zheng, ${ }^{3}$ Xinyi Zhang, ${ }^{4}$ Liang Wang, ${ }^{1,2}$ Yanfei Wang, \\ and Hao Wan ${ }^{1,2}$ \\ ${ }^{1}$ State Grid Shaanxi Electric Power Science Research Institute, Xi'an, China \\ ${ }^{2}$ State Grid (Xi'an) Environmental Technology Center Co., Ltd, Xi'an, China \\ ${ }^{3}$ State Grid Co., Ltd. DC Construction Branch, Beijing, China \\ ${ }^{4}$ State Grid Shaanxi Electric Power Xi'an Power Supply Company, Xi'an, China \\ ${ }^{5}$ Sichuan Hengchuang Tiandi Automation Equipment Co., Ltd., Chengdu, China \\ Correspondence should be addressed to Jian Wu; jwu.sxsp@yahoo.com
}

Received 16 July 2021; Revised 5 August 2021; Accepted 16 August 2021; Published 29 November 2021

Academic Editor: Bai Yuan Ding

Copyright $\odot 2021$ Lei Lei et al. This is an open access article distributed under the Creative Commons Attribution License, which permits unrestricted use, distribution, and reproduction in any medium, provided the original work is properly cited.

\begin{abstract}
Image analysis of power equipment has important practical significance for power-line inspection and maintenance. This paper proposes an image recognition method for power equipment based on multitask sparse representation. In the feature extraction stage, based on the two-dimensional (2D) random projection algorithm, multiple projection matrices are constructed to obtain the multilevel features of the image. In the classification process, considering that the image acquisition process will inevitably be affected by factors such as light conditions and noise interference, the proposed method uses the multitask compressive sensing algorithm (MtCS) to jointly represent multiple feature vectors to improve the accuracy and robustness of reconstruction. In the experiment, the images of three types of typical power equipment of insulators, transformers, and circuit breakers are classified. The correct recognition rate of the proposed method reaches $94.32 \%$. In addition, the proposed method can maintain strong robustness under the conditions of noise interference and partial occlusion, which further verifies its effectiveness.
\end{abstract}

\section{Introduction}

With the continuous increase of power equipment, traditional manual-line inspection and substation monitoring have been difficult to meet the actual requirements. In this context, a large number of power-line inspection equipment types based on helicopters, drones, and other computational platforms have been put into application [1-5]. These devices collect images of power equipment through optical and infrared sensors on themselves or nearby. Then, the image analysis and other technical means can be used to determine possible faults in the power equipment. Therefore, it is of great significance to carry out the analysis and interpretation of power equipment images. Image recognition of power equipment is one of the branches in the field of image analysis of power equipment. The basic idea is to classify the collected power equipment on the basis of the existing database, so as to provide a prerequisite for the targeted analysis of the special type of equipment. At present, there are still few studies in this field. In fact, the power equipment image recognition problem is similar to the traditional image-based target recognition problem and basically uses two stages of feature extraction and classifiers. In the process of feature extraction, according to the characteristics of power equipment images, geometric shape features, image gray distribution features, and local texture features are developed and employed [6-12]. Afterwards, an appropriate classifier is selected to process the extracted features and determine the corresponding category. Commonly used classifiers in image recognition of power equipment include support vector machines (SVMs) and sparse representationbased classification (SRC). In recent years, the deep learning models represented by the convolutional neural network $(\mathrm{CNN})$ have become a powerful tool in the field of image 
processing [13-16] and have also been widely used and verified in the image recognition of power equipment [17-21].

The rapid development of modern pattern recognition technology provides a large number of tools, which can be well used for reference in the recognition of power equipment images. In recent years, the compressive sensing (CS) technology has developed rapidly and widely used in the fields of signal processing, image analysis, and other relevant applications. Specifically, in the field of image recognition, $\mathrm{SRC}$ is a representative classifier based on the compressed sensing theory, which has been successfully applied in face recognition and remote sensing image recognition [22-24]. This paper makes optimizations in the feature extraction and classifier stages based on the CS theory and develops an image recognition method of power equipment. In the process of feature extraction, the two-dimensional (2D) random projection is used to reduce the dimensionality of the original images [25]. Through multiple random projection matrices, the characteristics of the image can be obtained from different aspects to achieve complementary enhancement. 2D random projection is an extension of traditional one-dimensional random projection, which can effectively maintain the structural characteristics of $2 \mathrm{D}$ signals such as images. At the same time, the algorithm inherits the advantages of random projection, which needs no prior parameters and has high computational efficiency. Through the comprehensive operation of multiple 2D random projection matrices, it can play a complementary role, so as to provide more comprehensive feature descriptions for the input images. In the classification stage, the multitask compressed sensing (MtCS) [26] is used to jointly reconstruct and analyze the multilevel projection features. MtCS is a typical multitask joint sparse representation algorithm, which can be used to analyze the internal correlation of multiple sparse representation tasks, so as to improve the accuracy of the solution [27-31]. In the field of target recognition, based on the solved sparse representation coefficients, the test samples can be reconstructed by different classes, so as to make decisions based on the reconstruction error. MtCS combines the principles of CS and Bayesian theory to obtain the largest a posteriori solution in the theoretical sense. In particular, due to the basic principles of CS and Bayesian solution, the algorithm can effectively overcome the influence of noise and other interference factors and can better handle the changes in lighting conditions, sensor noise, and other factors that may be encountered during the image acquisition process of power equipment. In order to verify the proposed method, images of insulators, transformers, and circuit breakers are used as basic samples in the experiments. And, noise interference and partial occlusion conditions are further constructed for testing. The experimental results show the effectiveness and robustness of the proposed method.

\section{2D Random Projection for Feature Extraction}

Traditionally, the projection features are extracted by first stacking the $2 \mathrm{D}$ image $X \in R^{n_{1} \times n_{2}}$ into a vector as
$x=\operatorname{vec}(X)$. Such operation actually benefits the processing afterwards but inevitably corrupts the structural information of the image. In order to maintain the structural properties of images, the $2 \mathrm{D}$ projection algorithm can be employed like $Y=A X B^{T}$, in which $Y \in R^{m_{1} \times m_{2}}$ denotes the resulted feature and $A \in R^{m_{1} \times n_{1}}$ and $B \in R^{m_{2} \times n_{2}}$ are the projection matrices with $m_{1} \ll n_{1}$ and $m_{2} \ll n_{2}$. The key in 2 D projection lies on the design of the projection matrices, which influences the validity of the final features.

According to the CS theory, the sparse signal can be well reconstructed by a small number of measurements. In a similar idea, the sparse matrix can be reconstructed by the low-dimensional matrix. In this sense, the CS theory provides a simple and effective way to extract features from $2 \mathrm{D}$ sparse matrices [25]. With the projection features $A \in R^{m_{1} \times n_{1}}$ and $B \in R^{m_{2} \times n_{2}}$, the process of feature extraction can be described as $Y=A X B^{T}$, where $X \in R^{n_{1} \times n_{2}}$ is the input image.

In order to guarantee the fidelity after feature extraction, the dimensions of the result $Y$, i.e., $m_{1}$ and $m_{2}$, should be properly chosen. According to [25], the requirements for $m_{1}$ and $m_{2}$ are as follows:

$$
\begin{aligned}
\|X\|_{0} & <\frac{\operatorname{spark}(A) \operatorname{spark}(B)}{4}, \\
\left(\ell^{0} \text { norm of each column of } X\right) & <\frac{\operatorname{spark}(A)}{2}, \\
\left(\ell^{0} \text { norm of each row of } X\right) & <\frac{\operatorname{spark}(B)}{2},
\end{aligned}
$$

where $\|X\|_{0}$ denotes the $\ell^{0}$ norm of $X$ and the spark of a matrix represents the minimal number of its columns which are linearly dependent. For the random projection matrices $A \in R^{m_{1} \times n_{1}}$ and $B \in R^{m_{2} \times n_{2}}$, they comply to spark $(A)=m_{1}+$ 1 and $\operatorname{spark}(B)=m_{2}+1$.

Owing to the merits of $2 \mathrm{D}$ random projection, this paper employs it for feature extraction of images of power equipment. Specially, multiple random projection matrices are developed to generate multiple feature vectors, which provide complementary descriptions of the target to be analyzed and classified.

\section{MtCS for Classification}

3.1. Basics of MtCS. It is easy to understand that the multiple measurements from the same source are statistically related like multichannel signals and multiview signatures [27-31]. MtCS is a multitask learning algorithm based on CS and Bayesian theory, which could consider the correlations of several related tasks to achieve high reconstruction precision.

Denote the $L$ measurements from the same source as $\left\{y_{i}\right\}_{i=1, \ldots, L}$; they are expressed as follows:

$$
y_{i}=A_{i} x_{i}+n_{i}
$$

where $A_{i} \in R^{N_{i} \times N}$ denotes the dictionary corresponding to $i$ th measurement and $n_{i}$ represents a zero-mean Gaussian process with the variance of $\xi_{0}$.

The likelihood function of $y_{i}$ is modeled as follows: 


$$
p\left(y_{i} \mid x_{i}, \xi_{0}\right)=\left(\frac{2 \pi}{\xi_{0}}\right)^{-N_{i} / 2} \exp \left(-\frac{\xi_{0}}{2}\left\|y_{i}-A_{i} x_{i}\right\|_{2}^{2}\right)
$$

The parameters $x_{i}(i=1,2, \ldots, L)$ are kept unchanged for all the $L$ tasks. The $L$ tasks are assumed to be statistically related as follows:

$$
p\left(y_{i} \mid \xi, \xi_{0}\right)=\prod_{j=1}^{N} N\left(x_{i, j} \mid 0, \xi_{j}^{-1} \xi_{0}^{-1}\right),
$$

where $x_{i, j}$ is the $j$ th element of $x_{i}$ and $\xi=\left[\xi_{j}, \ldots, \xi_{N}\right]^{T}$. The Gamma priors are put on the parameter $\xi_{0}$ as follows:

$$
p\left(\xi_{0} \mid a, b\right)=\operatorname{Gamma}\left(\xi_{0} \mid a, b\right) .
$$

With the choices of $\xi$ and $y_{i}$, the posterior density function (PDF) of $x_{i}$ can be calculated as follows:

$$
\begin{aligned}
p\left(x_{i} \mid y_{i}, \xi\right) & =\int p\left(x_{i} \mid y_{i}, \xi, \xi_{0}\right) p\left(\xi_{0} \mid a, b\right) \mathrm{d} \xi_{0} \\
& =\frac{\Gamma(a+N / 2)\left[1+\left(x_{i}-\mu_{i}\right)^{T} \sum_{i}^{-1}\left(x_{i}-\mu_{i}\right) / 2 b\right]^{-(a+N / 2)}}{\Gamma(a)(2 \pi b)^{N / 2}\left|\Sigma_{i}^{-1}\right|^{1 / 2}},
\end{aligned}
$$

where

$$
\begin{aligned}
& \mu_{i}=\Sigma_{i} A_{i}^{T} y_{i}, \\
& \Sigma_{i}=\left(A_{i}^{T} A_{i}+\Lambda\right)^{-1},
\end{aligned}
$$

with $\Lambda=\operatorname{diag}\left(\xi_{1}, \xi_{2}, \ldots, \xi_{N}\right)$.
The parameter $\xi$ can be estimated by searching the maximum of the marginal likelihood as follows:

$$
\begin{aligned}
L(\xi) & =\sum_{i=1}^{L} \log \left(y_{i} \mid \xi\right) \\
& =-\frac{1}{2} \sum_{i=1}^{L}\left[\left(N_{i}+2 a\right) \log \left(y_{i}^{T} B_{i}^{-1} y_{i}+2 b\right)+\log \left|B_{i}\right|\right]+\text { const },
\end{aligned}
$$

where $B_{i}=\mathrm{I}+A_{i} \Lambda^{-1} A_{i}^{T}$.

The dependence of $L(\xi)$ on $\xi_{j}$ and $B_{i}$ can be formulated as $B_{i}=B_{i,-j}+\xi_{j}^{-1} A_{i, j} A_{i, j}^{T}$ with $B_{i,-j}=I+\sum_{k \neq j} \xi_{k}^{-1} A_{i, k} A_{i, k}^{T}$. Therefore, $L(\xi)$ can be reformulated as follows:

$$
L(\xi)=L\left(\xi_{-j}\right)-\frac{1}{2} \sum_{i=1}^{L}\left[\log \left(\frac{1+s_{i, j}}{\xi_{j}}\right)+\left(N_{i}+2 a\right) \log \left(1-\frac{q_{i, j}^{2} / g_{i, j}}{\xi_{j}+s_{i, j}}\right)\right] \text {, }
$$

where $\xi_{-j}$ is obtained by removing the $j$ th component in $\xi$.

$$
\begin{aligned}
s_{i, j} & =A_{i, j}^{T} B_{i,-j}^{-1} A_{i, j}, q_{i, j} \\
& =A_{i, j}^{T} B_{i,-j}^{-1} y_{i} \text { and } g_{i, j} \\
& =y_{i}^{T} B_{i,-j}^{-1} y_{j}+2 b .
\end{aligned}
$$

To find the maximum of $L(\xi), L(\xi)$ is differentiated with respect to $\xi_{j}$. With the assumption of $\xi_{j} \ll s_{i, j}$, $\xi_{j}$ can be approximated as follows:

$$
\xi_{j} \approx \begin{cases}\frac{L}{\sum_{i=1}^{L}\left(\left(N_{i}+2 a\right) q_{i, j}^{2} / g_{i, j}-s_{i, j} / s_{i, j}\left(s_{i, j}-q_{i, j}^{2} / g_{i, j}\right)\right)}, & \text { if } \sum_{i=1}^{L} \frac{\left(N_{i}+2 a\right) q_{i, j}^{2} / g_{i, j}-s_{i, j}}{s_{i, j}\left(s_{i, j}-q_{i, j}^{2} / g_{i, j}\right)}>0, \\ \infty, & \text { otherwise. }\end{cases}
$$

Equation (11) controls the addition and deletion of $A_{i, j}$ from the signal representation. Then, $s_{i, j}, q_{i, j}$, and $g_{i, j}$ can be computed as follows:

$$
\begin{gathered}
s_{i, j}=\frac{\xi_{j} S_{i, j}}{\xi_{j}-S_{i, j}}, \\
q_{i, j}=\frac{\xi_{j} Q_{i, j}}{\xi_{j}-Q_{i, j}}, \\
g_{i, j}=G_{i}+\frac{Q_{i, j}^{2}}{\xi_{j}-S_{i, j}},
\end{gathered}
$$

with

$$
\begin{aligned}
S_{i, j} & =A_{i, j}^{T} A_{i, j}-A_{i, j}^{T} A_{i} \sum_{i} A_{i}^{T} A_{i, j}, \\
Q_{i, j} & =A_{i, j}^{T} y_{i}-A_{i, j}^{T} A_{i} \sum_{i} A_{i}^{T} y_{i}, G_{i}=y_{i}^{T} y_{i}-y_{i}^{T} A_{i} \sum_{i} A_{i}^{T} y_{i}+2 b .
\end{aligned}
$$

In equation (13), $A_{i}$ and $\Sigma_{i}$ only contain the basis vectors currently included in the model. With the sedimentations of $\Sigma_{i}$, the sparse representation coefficients can be solved. With the solutions of sparse coefficients corresponding to different tasks, the original input can be reconstructed class by class to calculate the reconstruction errors. Finally, by comparison of the reconstruction errors from different classes, the category of the input can be decided. 
3.2. Procedure of Target Recognition. Based on the above analysis, the basic process of the power equipment image recognition method designed in this paper is shown in Figure 1. First, multiple 2D random projection matrices are initialized for multilevel feature extraction. For all training samples, a corresponding dictionary is constructed through different $2 \mathrm{D}$ random projection matrices to form a multifeature dictionary. The test sample uses the same random projection matrices to obtain the multilevel feature vectors. Then, based on the multifeature dictionary, MtCS is used to characterize multiple feature vectors of the test sample and the sparse representation coefficients corresponding to different features are calculated. Finally, the decision is made based on the reconstruction errors of the test sample corresponding to different training classes. The proposed method shows a certain degree of randomness in the construction of $2 \mathrm{D}$ random projections, so as to ensure the complementarity between them. In the classification stage, the maximum posterior strategy adopted by MtCS can well overcome the interference caused by nuisance conditions such as noise and occlusion, so as to ensure the reliability of the final decision.

\section{Experiments and Discussion}

4.1. Description of the Dataset. In order to test the performance of the proposed method, this paper uses three types of power equipment, i.e., insulators, transformers, and circuit breakers, for experiments, which are common in power systems. 2000 images of each of the three types of equipment are collected and used. All these images are adjusted to sizes of 400 pixels $\times 400$ pixels by means of preprocessing. 1400 images of each of the three types of targets are randomly selected as the training samples, and the remaining 600 images are used as the test samples. As a comparison, this paper selects several types of existing relevant methods to conduct experiments at the same time, including the method based on the region moments in [11] (denoted as Region moment), the method using SRC in [5] (denoted as SRC), the method based on SVM in [8] (denoted as SVM), and the method using CNN in [21] (denoted as CNN).

In the following, the original samples are first tested as a preliminary validation. Later, the nuisance conditions, which may occur in the actual applications, are considered including noise interference and occlusion. The performance of all the methods is comprehensively investigated under the three conditions to reach the final evaluation of the proposed method.

4.2. Performance on Original Samples. At first, we use the proposed method to classify the original test samples of three types of equipment. Table 1 shows the recognition results of the three types of equipment achieved by the proposed method. It can be seen that the recognition rate of the three types of equipment has reached more than $90 \%$, and the average recognition rate is calculated to $92.3 \%$. This result shows the effectiveness of the proposed method for image recognition of power equipment. Table 2 compares the average recognition rates of different methods. The performance of the proposed method is better than of others, which proves its superior performance. Specifically, compared with the SRC method, this paper extends the single sparse representation problem to a multitask one and combines multilevel $2 \mathrm{D}$ random projection features to provide a more adequate discrimination basis for classification decision. Therefore, the recognition result of the proposed method is greatly improved compared with the SRC method. The recognition performance of the CNN method ranks second in this case, only lower than the proposed method. For the original samples, the test samples can maintain high correlations with the training samples. At this time, the trained classification network can maintain strong adaptability to the test samples. The method based on regional moment features has poor performance under the current condition, mainly because there may be certain errors in the process of regional feature extraction, which are passed to the classification stage and cause the decrease of the recognition accuracy.

4.3. Performance on Noisy Samples. Since noise interference is inevitable in the actual image acquisition process, it is necessary to investigate the recognition performance of the proposed method under noise interference conditions. In this experiment, we first add different degrees of Gaussian white noise to the original test samples [32] and then test the recognition performance of different methods for noise samples. Figure 2 shows the average recognition rate curves of different methods as the signal-to-noise ratio (SNR) changes. It can be seen that the proposed method maintains the best recognition performance under different SNRs, indicating its robustness to noise interference. As analyzed above, both $2 \mathrm{D}$ random projection and MtCS are based on the basic theory of CS and have good adaptability to noise influences. At the same time, Bayesian estimation is introduced into MtCS, which can further enhance the robustness of the classification process against noise interference. Compared with the condition of the original samples, the performance of the CNN and SVM methods degrades the most significantly. Taking the CNN as an example, as the noise level of the test samples continues to increase, its similarity with the training samples decreases. As a result, the trained classification network has poor adaptability to those test samples, resulting in a decrease in the recognition accuracy. The overall robustness of the method based on the regional moment feature under noise interference is second only to the proposed method, because the regional feature is relatively insensitive to noise interference. Even under noise pollution conditions, the area characteristics of the target can generally be well maintained, so it can maintain good performance under noise interference conditions. The SRC method has some advantages over the CNN and SVM, which further reflects the robustness of the CS principle to noise interference.

4.4. Performance on Occluded Samples. In the process of collecting images of power equipment, occlusion and other situations inevitably occur, resulting in partial occlusions in the acquired images. To test the adaptability of the proposed method to occlusion conditions, this paper constructs occluded samples based on the original test set and obtains 


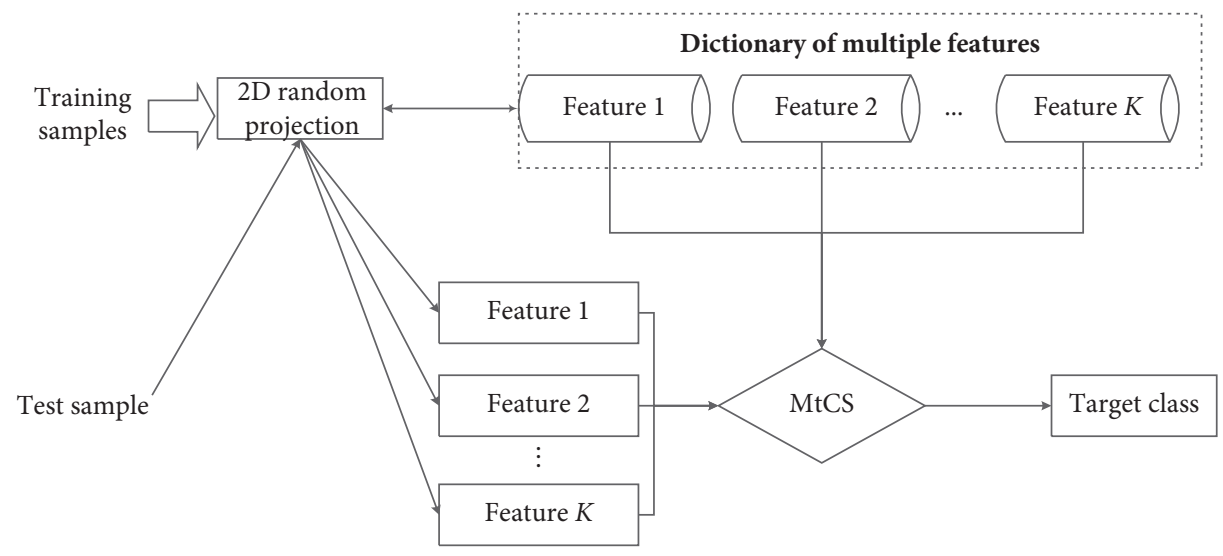

FIgURE 1: Procedure of recognition via electric equipment based on 2D random projection and MtCS.

TABLE 1: The recognition results of the three types of power equipment achieved by the proposed method.

\begin{tabular}{lcccc}
\hline Class & Recognition result & Circuit breakers & Recognition rate (\%) \\
\hline Insulators & Insulators & Transformers & 27 & 92.00 \\
Transformers & 552 & 580 & 11 & 96.67 \\
Circuit breakers & 9 & 13 & 567 & 94.50 \\
Average recognition rate (\%) & 10 & \multicolumn{2}{c}{94.32} & \\
\hline
\end{tabular}

TABLE 2: Comparison of performance of different methods on original test samples.

\begin{tabular}{lcccrr}
\hline Method type & Proposed & Region moment & SVM & SRC & CNN \\
\hline Average recognition rate (\%) & 94.32 & 91.87 & 92.13 & 92.42 & 93.54 \\
\hline
\end{tabular}

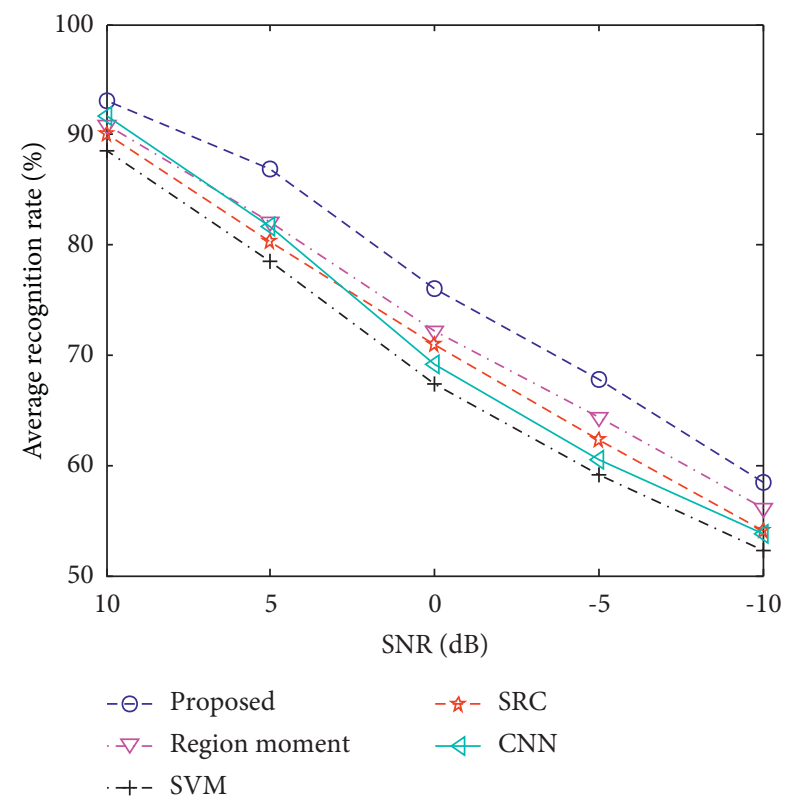

FiguRE 2: Comparison of performance of different methods on noisy samples. 


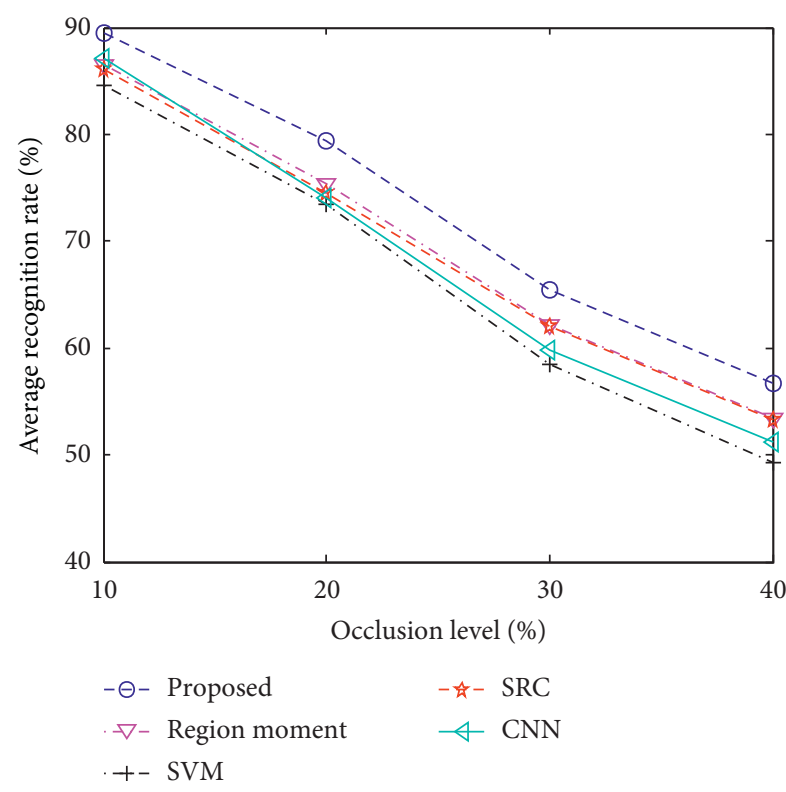

FIGURE 3: Comparison of performance of different methods on occluded samples.

different test sets with occlusion levels of $10 \%, 20 \%, 30 \%$, and $40 \%$. Afterwards, all the methods are used to classify the test sets with different occlusion conditions, and the statistical results are shown in Figure 3. It can be seen that the proposed method can achieve higher performance than the comparison methods at different occlusion levels, showing its robustness to partial occlusion. The multilevel $2 \mathrm{D}$ random projection features have good complementarity, so they play a positive role in the recognition problem under occlusion conditions. At the same time, the Bayesian estimation used in MtCS can be solved in a maximum posterior manner, so as to obtain a theoretical optimal decision. Similar to the case of noise interference, the performance of the SRC method is better than that of the SVM and CNN methods, which reflects that the principle of CS is also adaptable to occlusion situations. Therefore, the proposed method combines the advantages of these two tools to effectively improve the adaptability to occlusion situations.

\section{Conclusion}

For the problem of power equipment image recognition, this paper proposes a method based on $2 \mathrm{D}$ random projection and MtCS. The multilevel feature vectors of the power equipment image are obtained by $2 \mathrm{D}$ random projection, which have good complementarity. MtCS has good noise robustness and anti-interference performance and can robustly solve sparse representation coefficients under more complex conditions. By combining the advantages of the two algorithms, the adaptability of the recognition method to various scenarios can be improved. Experiments are carried out based on the samples of three typical power equipment: insulators, transformers, and circuit breakers. The results show that the proposed method is effective for the problem and has stronger robustness than other methods under conditions like noise interference and partial occlusions.

\section{Data Availability}

Our experiment data are all collected from the Internet, and they are permitted for public use.

\section{Conflicts of Interest}

The authors declare that they have no conflicts of interest.

\section{References}

[1] C. Guo, M. Ren, C. Xia, M. Dong, and B. Wang, "Fault diagnosis of power equipment based on infrared image analysis," in Proceedings of the IEEE International Conference on Advances in Electrical Engineering and Computer Applications (AEECA), pp. 659-663, Dalian, China, August 2020.

[2] J. Ma, K. Qian, X. Zhang, and X. Ma, "Weakly supervised instance segmentation of electrical equipment based on RGBT automatic annotation," IEEE Transactions on Instrumentation and Measurement, vol. 69, no. 12, pp. 9720-9731, 2020.

[3] G. C. Stone, "Partial discharge diagnostics and electrical equipment insulation condition assessment," IEEE Transactions on Dielectrics and Electrical Insulation, vol. 12, no. 5, pp. 891-903, 2005.

[4] T. Liu, "Development and application of electrical equipment intelligent detection management system," in Proceedings of the 5th International Conference on Smart Grid and Electrical Automation (ICSGEA), pp. 70-73, Zhangjiajie, China, June 2020.

[5] C.-F. Lai, W.-C. Chien, L. T. Yang, and W. Qiang, "LSTM and edge computing for big data feature recognition of industrial electrical equipment," IEEE Transactions on Industrial Informatics, vol. 15, no. 4, pp. 2469-2477, 2019.

[6] D. Ming, B. Wang, M. Ren et al., "Joint visualization diagnosis of outdoor insulation status with optical and acoustical detections," IEEE Transactions on Power Delivery, vol. 34, no. 4, pp. 1221-1229, 2018.

[7] X. Xiong, S. Xu, W. Wu, D. Tu, J. Zhang, and Z. Wei, "Identification of electrical equipment based on faster LSTMCNN network," in Proceedings of the IEEE International Conference on Networking, Sensing and Control (ICNSC), pp. 1-6, Nanjing, China, November 2020.

[8] L. Jin, J. Ai, Z. Tian, and Y. Zhang, "Detection of polluted insulators using the information fusion of multispectral images," IEEE Transactions on Dielectrics and Electrical Insulation, vol. 24, no. 6, pp. 3530-3538, 2017.

[9] L. Li, J. Yong, and W. Xu, "On-line cable condition monitoring using natural power disturbances," IEEE Transactions on Power Delivery, vol. 34, no. 4, pp. 1242-1250, 2019.

[10] J. Wang, J. Wang, J. Shao, and J. Li, "Image recognition of icing thickness on power transmission lines based on a least squares hough transform," Energies, vol. 10, no. 4, p. 415, 2017.

[11] Y. Yin, Z. Meng, and S. Li, "Feature extraction and image recognition for the electrical symbols based on Zernike moment," in Proceedings of the IEEE 2nd Advanced Information Technology, Electronic and Automation Control Conference (IAEAC), pp. 1031-1035, Chongqing, China, March 2017.

[12] Z. Zhao, G. Xu, and Y. Qi, "Representation of binary feature pooling for detection of insulator strings in infrared images," 
IEEE Transactions on Dielectrics and Electrical Insulation, vol. 23, no. 5, pp. 2858-2866, 2016.

[13] A. Krizhevsky, I. Sutskever, and G. E. Hinton, "Imagenet classification with deep convolutional neural networks," Proc NIPS, vol. 2, pp. 1096-1105, 2012.

[14] C. Szegedu, W. Liu, Y. Jia et al., "Going deeper with convolutions," in Proceedings of the2015 IEEE Conference on Computer Vision and Pattern Recognition (CVPR), pp. 1-9, Boston, MA, USA, June 2015.

[15] K. He, X. Zhang, S. Ren et al., "Deep residual learning for image recognition," in Proceedings of the 2015 IEEE Conference on Computer Vision and Pattern Recognition (CVPR), pp. 770-778, Las Vegas, NV, USA, June 2016.

[16] X. X. Zhu, D. Tuia, L. Mou et al., "Deep learning in remote sensing: a comprehensive review and list of resources," IEEE Geoscience and Remote Sensing Magazine, vol. 5, no. 4, pp. 8-36, 2017.

[17] X. Gong, Q. Yao, M. Wang, and Y. Lin, “A deep learning approach for oriented electrical equipment detection in thermal images," IEEE Access, vol. 6, Article ID 41590, 2018.

[18] C. Yeung, "Image Recognition Algorithm of Electrical Engineering Equipment Based on Machine Learning Method," in Proceedings of the IEEE International Conference on Power Electronics, Computer Applications, pp. 711-714, Shenyang, China, January 2021.

[19] Y. Xia, J. Lu, H. Li, and H. Xu, “A Deep Learning Based Image Recognition and Processing Model for Electric Equipment Inspection," in Proceedings of the 2018 2nd IEEE Conference on Energy Internet and Energy System Integration (EI2), pp. 1-6, Beijing, China, October 2018.

[20] X. Siheng, Y. Liu, R. Xu et al., "Power equipment recognition method based on mask R-CNN and bayesian context network," in Proceedings of the IEEE Power \& Energy Society General Meeting (PESGM), pp. 1-5, Montreal, Canada, August 2020.

[21] A. Jiang, N. Yan, B. Shen, C. Gu, H. Zhu, and H. Huang, "Research on infrared image recognition method of power equipment based on deep learning," in Proceedings of the IEEE International Conference on High Voltage Engineering and Application (ICHVE), pp. 1-4, Beijing, China, September 2020.

[22] J. Wright, A. Y. Yang, A. Ganesh, S. S. Sastry, and Y. Yi Ma, "Robust face recognition via sparse representation," IEEE Transactions on Pattern Analysis and Machine Intelligence, vol. 31, no. 2, pp. 210-227, 2009.

[23] J. J. Thiagaraianm, K. N. Ramamurthy, P. Knee et al., "Sparse representations for automatic target classification in SAR images," in Proceedings of the 2010 4th International Symposium on Communications, Control and Signal Processing (ISCCSP), pp. 1-4, Limassol, Cyprus, March 2010.

[24] H. Song, K. Ji, Y. Zhang, X. Xing, and H. Zou, "Sparse representation-based SAR image target classification on the 10-class MSTAR data set," Applied Sciences, vol. 6, no. 1, p. 26, 2016.

[25] B. Ding, G. Wen, F. Ye, X. Huang, and X. Yang, "Feature extraction based on $2 \mathrm{D}$ compressive sensing for SAR automatic target recognition," in Proceedings of the 11th European Conference on Antennas and Propagation (EUCAP), pp. 1219-1223, Paris, France, March 2017.

[26] S. Ji, D. Dunson, and L. Carin, "Multitask compressive sensing," IEEE Transactions on Signal Processing, vol. 57, no. 1, pp. 92-106, 2009.

[27] Q. Qisong Wu, Y. D. Zhang, M. G. Amin, and B. Himed, "Multi-task bayesian compressive sensing exploiting intra- task dependency," IEEE Signal Processing Letters, vol. 22, no. 4, pp. 430-434, 2015.

[28] J. A. Tropp, A. C. Gilbert, and M. J. Strauss, "Algorithms for simultaneous sparse approximation. Part II: c," Signal Processing, vol. 86, no. 3, pp. 589-602, 2006.

[29] H. Zhang, N. M. Nasrabadi, Y. Zhang, and T. S. Huang, "Multi-view automatic target recognition using joint sparse representation," IEEE Transactions on Aerospace and Electronic Systems, vol. 48, no. 3, pp. 2481-2497, 2012.

[30] B. Ding and G. Wen, "Exploiting multi-view SAR images for robust target recognition," Remote Sensing, vol. 9, no. 11, p. 1150, 2017.

[31] S. Liu and J. Yang, "Target recognition in synthetic aperture radar images via joint multifeature decision fusion," Journal of Applied Remote Sensing, vol. 12, no. 1, Article ID 016012, 2018.

[32] X. Zhang, "Noise-robust target recognition of SAR images based on attribute scattering center matching," Remote Sensing Letters, vol. 10, no. 2, pp. 186-194, 2019. 